\title{
Brain abscess with Clostridium cadaveris and Clostridium sporogenes
}

\author{
Rakesh Parakadavathu ${ }^{1}$, Mohammed Abu Khathab ${ }^{1}$, Muna Al-Maslamani ${ }^{1}$, Arun P. Nair ${ }^{1}$, Manoj K \\ Varghese ${ }^{1}$, Anand Deshmukh ${ }^{2}$, Aisha Larem ${ }^{3}$, Hassan Haider ${ }^{3}$, Talal Alrabayah ${ }^{4}$, Adnan Khan $^{4}$, Faraj \\ Howady ${ }^{1}$ \\ ${ }^{1}$ Department of Microbiology, Hamad Medical Corporation, Qatar \\ ${ }^{2}$ Department of Microbiology, Hamad Medical Corporation, Qatar \\ ${ }^{3}$ Department of ENT, Hamad Medical Corporation, Qatar \\ ${ }^{4}$ Department of Neurosurgery, Hamad Medical Corporation, Qatar
}

\begin{abstract}
Brain abscess is a collection of pus in the parenchyma of brain. Most common infecting organisms are streptococci, staphylococci and anaerobes, depending upon the source from which it originates. Clostridium cadaveris and Clostridium sporogenes are common organisms found in the gastrointestinal tract of humans, rarely producing disease. Few cases of bacteremia and abscesses are reported with these organisms. We hereby report the first case of brain abscess with $C$ cadaveris and $C$. sporogenes in a young male with good outcome by surgical and medical management. J Microbiol Infect Dis 2019; 9(3):129-132.
\end{abstract}

Keywords: Clostridium cadaveris, Clostridium sporogenes, Brain abscess

\section{INTRODUCTION}

Clostridium species are ubiquitous, some of them commensals of the intestinal flora and can lead illness like self-limiting food poisoning to life threatening gas gangrene or tetanus. $C$ cadaveris and $C$. sporogenesis infections are extremely rare, the former being the predominant bacteria during putrification of dead bodies. Here we describe a case of brain abscess from which $C$ cadaveris and $C$. sporogenesis were isolated with good treatment outcome.

\section{CASE}

21 year old male was admitted to our hospital with history of diffuse headache, low grade fever for two weeks and right ear discharge with pain for one week. Four days before admission, he developed nausea and vomited multiple times.

There was history of right lower molar tooth extraction done 35 days back in a private clinic. Patient is not known to have diabetes, systemic hypertension or valvular heart disease. He was Nepalese by origin and was residing in Qatar for the last nine months. He reported no smoking, alcohol use or intravenous drug abuse.

On examination he was alert and co-operative, not in any distress with stable vital signs. Right ear examination showed patent ear canal, yellowish discharge over tympanic membrane and attic retraction pocket with cholesteatoma; nervous system examination was within normal limits.

Blood counts, routine chemistry and chest X-ray were normal. Emergency non-contrast CT scan of the head showed hypodense lesions in right superior cerebellum at the posterior and anterior aspect with mild peri-lesional edema, significant mass effect and obstructive hydrocephalus; features suggestive of cerebellar abscess which was confirmed by MRI (Figure 1)

Patient was planned for immediate aspiration of the abscess. He was empirically given intravenous ceftriaxone $2 \mathrm{~g}$ IV $\mathrm{q} 12 \mathrm{~h}$, vancomycin $1 \mathrm{~g} \mathrm{IV} \mathrm{q} 12 \mathrm{~h}$ and metronidazole 750 $\mathrm{mg}$ IV q8h. He underwent drainage of right cerebellar abscess through suboccipital paramedian burr hole, navigation guided aspiration with external ventricular drain

Correspondence: Dr. Rakesh Parakadavathu, Department of Infectious Diseases, Hamad Medical Corporation, Doha, Qatar

E-mail: tparakadavathu@hamad.qa

Received: 16 January 2019 Accepted: 27 June 2019

Copyright (C JMID / Journal of Microbiology and Infectious Diseases 2019, All rights reserved 
insertion, right cortical mastoidectomy and cholesteatoma removal from mastoid and middle ear. Eight $\mathrm{ml}$ of frank pus was aspirated. Gram stain of the aspirate was obtained (Figure 2). There was profuse growth of Clostridium cadaveris, Clostridium sporogenes and Peptostreptococcus species as identified by MALDI-TOF machine.

Vancomycin was stopped and ceftriaxone and metronidazole was continued. His ear discharge grew Pseudomonas aeruginosa that was sensitive to all antibiotics and hence final regimen was changed to cefepime and metronidazole. Repeat MRI scan after 2 weeks showed resolution of smaller abscesses and reduction in size of the biggest one. He showed good clinical improvement and continued to be asymptomatic with no signs of relapse at 2 months follow up.

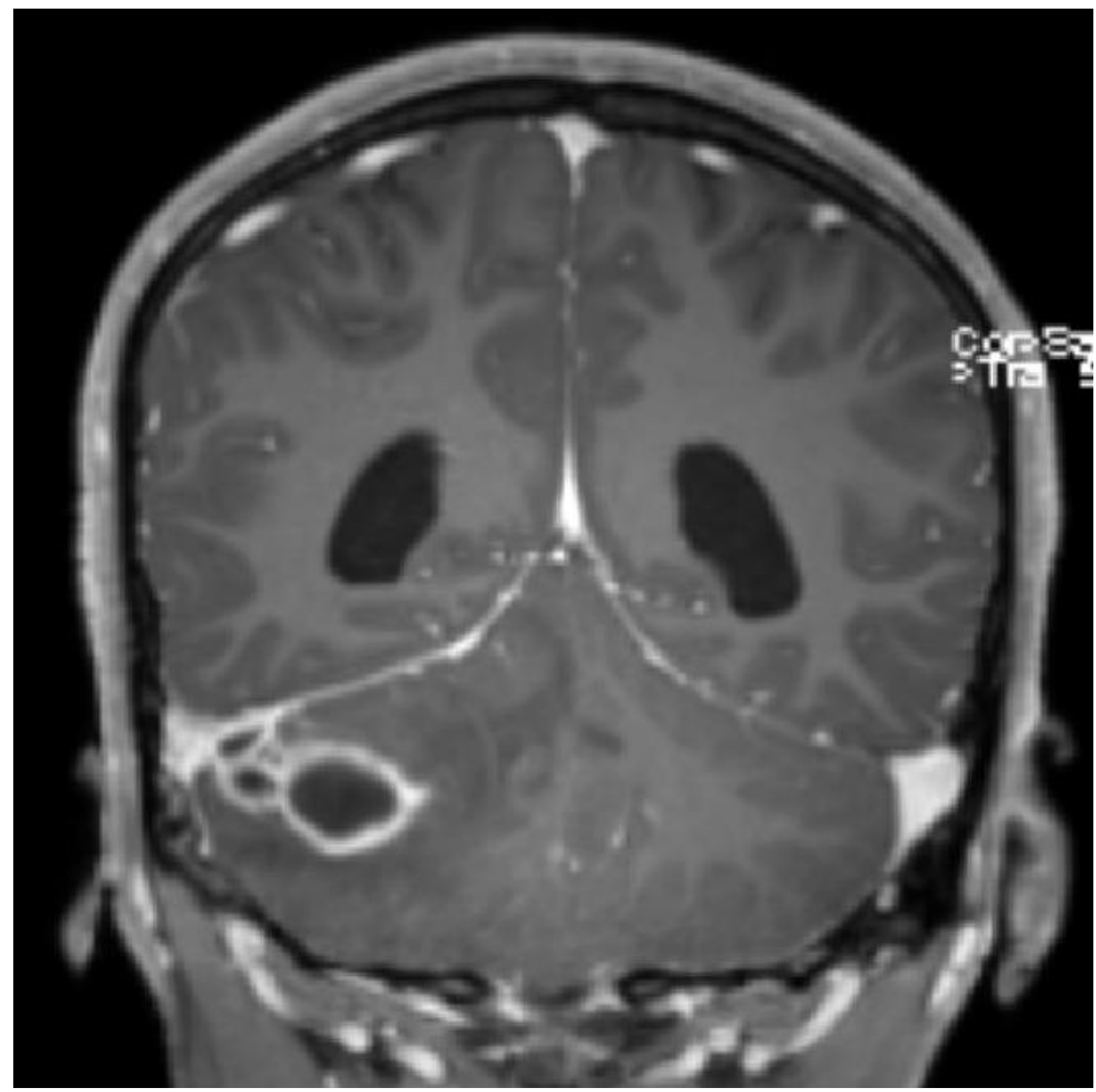

Figure 1. MRI of head showing right ear cholesteatoma with right multi focal cerebellar hemisphere abscesses about $3 \times 2,2 \times 1$, and $1 \mathrm{~cm}$ diameter in cross section, with marginal enhancement and central diffusion restriction, associated with significant right cerebellar hemispheric swelling, mid line shift, encroachment on the fourth ventricle and mild ventriculomegaly with venous thrombosis involving right sigmoid and distal right transverse dural sinuses. 


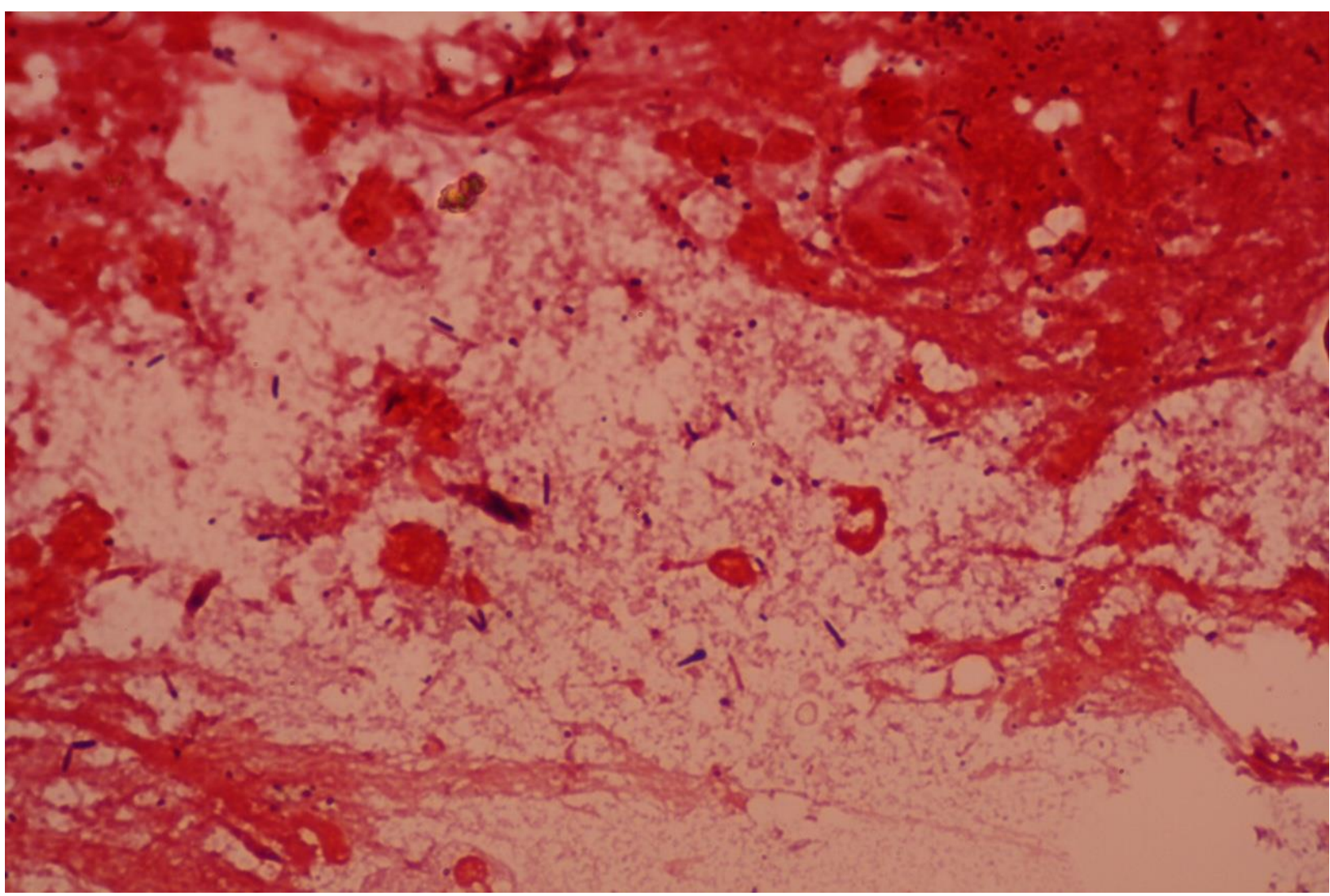

Figure 2. Pus aspirate showing profuse polymorphonuclear leukocytes, gram positive bacilli with terminal spores, gram positive cocci and scanty gram negative bacilli. HEx100

Infections of ear, nose and throat remain the most frequent etiology for brain abscess [1]. The causative organism is variable, depends on the patient age, predisposing condition and immune status. Commonly isolated organisms include staphylococcus, streptococcus, bacteroides and peptostreptococcus [2].

The genus Clostridium consist of more than 200 species, some of them are commensals of the gut microflora and can produce variety of infections in humans and animals. The organisms are ubiquitous in environment. The commonly disease producing species include $\mathrm{C}$. tetani, C. botulinum, C. perfringens, C. novyi, C. septicum, C. histolyticum, C. perfringens and C. difficile. Clostridial infections can result in food poisoning, necrotizing enterocolitis, skin and soft tissue infections and gas gangrene.

$C$ cadaveris is commonly found in the gastrointestinal tract of humans and is the predominant bacteria during decomposition of dead bodies. Very rarely it produces infection and only nine case reports are published so far.
Majority of the reported cases were associated with bacteremia secondary to bowel perforation and other sources being pleura and joint. $C$ cadaveris generally remain sensitive to most of the antibiotics [3]. Only one case of central nervous system infection- meningitis- with $C$ cadaveris is reported [4].

C. sporogenes was first described by Metchnikoff in 1908, who isolated it from the gastrointestinal tracts of normal individuals and patients with chronic colitis [5]. It is capable of producing a hemorrhagic toxin which contributes to the virulence [6]. C. sporogenes rarely produce disease in humans and majority of reported cases are bacteremia in cancer patients on chemotherapy. One case of postoperative septic arthritis [7] and two cases of empyma thorax $[8,9]$ are also reported.

Peptostreptococcus is one of the well described bacteria associated with brain abscess [10]. This is the first reported case of brain abscess with $C$ cadaveris and C. sporogenes. Both strains didn't show resistance to antibiotics except 
C.sporogens which showed intermediate sensitivity towards clindamycin.

Conclusions

Our case is the first reported case of brain abscess from which $C$ cadaveris and $C$. sporogenes were isolated. Polymicrobial infection were reported in majority of the cases of brain abscess and in one series it showed correlation with the bacteria in middle ear or paranasal sinus in only $38 \%$, nullifying the results of ENT samples for choosing antibiotic regimens [1]. We believe that the Psuedomonas which grown form the ear discharge was not contributing to the brain abscesses but was responsible for the mastoiditis and the origin of the bacteria causing brain abscess is from the oral flora related to tooth extraction done previously. Anaerobes continues to be one of the common causes of brain abscesses and the treating physicians should be well aware of this in order to include medications to cover it in the empirical regimen. Isolating the precise organism is of clinical importance as antibiotics can be tailored and narrowed.

\section{ACKNOWLEDGMENTS}

The authors would like to thank all the staff of Hamad Medical Corporation, Qatar, involved in the patient care.

Conflict of Interest: There is no conflict of interest involved in this case. Financial Disclosure: There is no financial support in this case report

\section{REFERENCES}

1. Couloigner V, Sterkers $O$, Redondo A, Rey A. Brain Abscesses of Ear, Nose, and Throat Origin: Comparison between Otogenic and Sinogenic Etiologies. Skull Base Surg. 1998; 8(4):163-168.

2. Muzumdar D, Jhawar S, Goel A, et al. Brain abscess: An overview. Int J Surg. 2011; 9(2):13644.

3. Pfaff C, Droege C, Magner M, Schrager J. Clostridium cadaveris bacteremia in a critically ill surgical patient with septic shock after total abdominal colectomy : a case report and review of literature. 2016; 2(1):1-4.

4. Fernández-López A, Jiménez-Mejías ME, GarcíaCuriel A, Palomino Nicás J. Meningitis caused by Clostridium cadaveris. Med Clin (Barc) 1991; 16;97(17):679.
5. Princewill TJT. Differences in Colony Morphology and Carbohydrate Fermentation of Clostridium sporogenes. Microbiology. 1978; 108(2):315-319.

6. Hara-kudo Y, Ogura A, Noguchi Y, Kumagai S. Characteristics of toxicity and haemorrhagic toxin produced by Clostridium sporogenes in various animals and cultured cells. J Med Microbiol 1997; $1 ; 46(4): 270-5$.

7. Inkster T, Cordina C, Siegmeth A. Septic arthritis following anterior cruciate ligament reconstruction secondary to Clostridium sporogenes; a rare clinical pathogen. J Clin Pathol 2011; 1;64(9):820821.

8. Corbett CE, Wall BM, Cohen M. Case report: empyema with hydropneumothorax and bacteremia caused by Clostridium sporogenes. Am J Med Sci 1996; 312(5):242-5.

9. Malmborg AS, Rylander M, Selander H. Primary thoracic empyema caused by Clostridium sporogenes. Scand J Infect Dis 1970;n2(2):155156.

10. Aras $Y$, Sabanci PA, Izgi N, et al. Surgery for pyogenic brain abscess over 30 years: Evaluation of the roles of aspiration and craniotomy. Turk Neurosurg 2016; 26(1):39-47. 